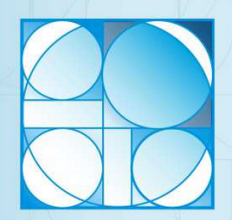

$2^{\circ}$ Prêmio Nacional de Ensino de Bioquímica e Biologia Molecular

Sociedade Brasileira de Bioquímica

e Biologia Molecular - SBBq
Journal of Biochemistry Education

Revista de Ensino de Bioquímica

Revista de Enseñanza de Bioquímica

Q4. Sociedade Brasileira de Bioguimica

ISSN: 2318-8790

ISSN (until 2012): 1677-2318

\title{
Applying a Design-Based Research Framework in the Production of Web Content to Disseminate the Remote Laboratory Uses and Development.
}

\author{
Oliveira, P.P.T. ${ }^{1}$; Galembeck, E. ${ }^{1}$
}

${ }^{1}$ Dep. de Bioquímica e Biologia Tecidual, IB, UNICAMP, SP, Brazil

INTRODUCTION. The adoption of teaching methods that highlight student's autonomy is a long term process and requires students to be involved with hands-on activities such as lab classes. Lab activities can vary in methodological approaches from demonstration to the authentic research, and requires the use of equipment, reagents, and time. A lot of low-cost experiments alternatives have been developed as an alternative to the expensive equipment not always available in didactic labs. The availability of low-cost microcontrollers and sensors has made possible not only having students do run experiments but also having them to build equipment to be used in their investigative labs. It is also possible to build equipment to be controlled over the internet and to build low-cost remote labs able to provides a real experience of controlling experiment online. OBJECTIVE. This work aims the production of a series of informative and explanatory videos about the use and development of low-cost equipment for remote operation, and to analyze the impact this strategy in biochemistry education, and knowledge dissemination. MATERIALS AND METHODS. Equipment and experiments already developed and in operation have been the starting point to produce a videos series for YouTube. The whole process is structured as Design-Based Research framework, which involves developing iterations of the material, each one incorporating changes based on the feedback taken about the source material. RESULTS AND DISCUSSION. We have produced 12 videos that have been released weekly on our YouTube channel. The topics covered so far are titration, photosynthesis and microscopic diversity. All videos are linked to a survey, which will serve to collect data regarding the utility and quality of the videos, reproducibility of the experiments, and demographic data from the viewers. CONCLUSION. The first two videos released have received positive feedback and a reasonable number of views.

Keywords: DBR, remote laboratory, education 